\title{
Programa de Residência Multiprofissional em Saúde: a percepção dos residentes de Saúde da Família
}

\author{
Multiprofessional Health Residency Program: the \\ perception of the residents of Family Health
}

\author{
Ana Paula Nascimento de Lima' (D), Ila landara Araújo de Souza' (D), \\ Lays Ariane Teixeira dos Santos', Biatriz Araújo Cardoso' (i) \\ 'Universidade do Estado do Pará, Belém (PA), Brasil.
}

\begin{abstract}
RESUMO
Introdução: A Residência Multiprofissional em Saúde (RMS), modalidade de ensino em serviço, se constitui como uma das estratégias potenciais para repensar o processo de formação e desenvolvimento dos profissionais de saúde, ao passo que assegura o desenvolvimento dos trabalhadores e instituições de saúde por meio de suas políticas. Com a RMS, os residentes têm diversas oportunidades de aprimoramento teórico e prático, o que pode possibilitar a reflexão crítica de várias ações e aspectos, que emergem a partir das situações vividas durante esse processo de formação. Objetivo: Analisar a percepção dos residentes de Saúde da Família acerca do Programa de Residência Multiprofissional em Saúde (RMS) da Universidade do Estado do Pará (UEPA). Métodos: A pesquisa foi do tipo observacional transversal, de caráter quanti-qualitativo, realizada com 10 dos 12 residentes integrantes do Programa em questão e do segundo ano (R2). Os voluntários foram submetidos a um questionário (perfil dos entrevistados) e uma entrevista semiestruturada contendo cinco questões norteadoras. Para os depoimentos, foi empregada a técnica de Análise de Conteúdo, onde seis categorias emergiram: a Residência Multiprofissional em Saúde; o papel dos Residentes; a Prática Multiprofissional na Residência; Atividades Curriculares da Residência; a Atuação dos Preceptores; os Aspectos Positivos e Negativos da Residência em Saúde da Família. Resultados: Os resultados sugeriram em suma, sobre os aspectos assertivos e desfavoráveis que concernem a RMS. Entre eles, as fragilidades na inserção dos residentes nos serviços de saúde e potencialidades na presença de diferentes formações profissionais. Conclusão: O estudo demonstrou que apesar de todos os avanços expostos através dos relatos descritos, a Residência Multiprofissional em Saúde da Família constitui um projeto em construção, que precisa de ações e mudanças para acontecer na prática, no cotidiano dos serviços, em conjunto com todos os atores envolvidos neste Programa.
\end{abstract}

\section{CORRESPONDÊNCIA}

Ana Paula Nascimento de Lima Universidade do Estado do Pará TV. Perebebuí, 2623, Marco, CEP 66087-662, Belém (PA), Brasil paula.nlima@hotmail.com

\section{FONTE DE FINANCIAMENTO}

Nenhuma.

\section{CONFLITO DE INTERESSE}

Os autores declararam não

haver conflitos de interesse.

O estudo foi realizado na Universidade do Estado do Pará, Belém (PA), Brasil.

\section{ABSTRACT}

Introduction: The Multiprofessional Health Residency (RMS), an in-service teaching modality, is one of the potential strategies to rethink the process of training and development of health professionals, while ensuring the development of health workers and institutions through Their policies. With RMS, residents have several opportunities for theoretical and practical improvement, which may allow the critical reflection of various actions and aspects that emerge from the situations lived during this formation process. Objective: To analyze the perception of the residents of Family Health about the Program of Multiprofessional Residency in Health (RMS) of the University of the State of Pará (UEPA). Methods: The research was a cross-sectional, quantitative-qualitative study carried out with 10 of the 12 residents of the Program in question and of the second year (R2). The volunteers were submitted to a questionnaire (profile of the interviewees) and a semi-structured interview containing five guiding questions. For the testimonies, the technique of Content Analysis was employed, where six categories emerged: the Multiprofessional Residency in Health; The role of Residents; The Multiprofessional Practice in the Residence; Curricular Activities of the Residence; The Performance of the Preceptors; The Positive and Negative Aspects of Family Health Residence. Results: The results suggested, in short, the assertive and unfavorable aspects that concern RMS. Among them, the weaknesses in the insertion of residents in health services and potentialities in the presence of professionals from different areas. Conclusion: The study demonstrated that despite all the advances exposed through the described reports, the Multiprofessional Residency in Family Health is a project under 
construction, which requires actions and changes to happen in practice, in the daily life of services, together with all the actors involved in this Program.

KEYWORDS: Unified Health System. Education, continuing. Family Health.

\section{Introdução}

O Sistema Único de Saúde (SUS) foi instituído no Brasil por meio da Constituição de 1988, na qual se afirmou a necessidade de promover a saúde como direito fundamental de cidadania ${ }^{1}$. A partir do conceito explanado no Art. 196 da Constituição Federal, onde define-se que: "A saúde é direito de todos e dever do Estado, garantido mediante políticas sociais e econômicas que visem à redução do risco de doença e de outros agravos e ao acesso universal e igualitário às ações e serviços para a promoção, proteção e recuperação [...]" (p.118); e regulamentado por meio das Leis Federais 8080/90 e 8142/90, a criação e implantação do SUS promoveu um novo direcionamento para as políticas públicas de saúde 2 .

Assim, dentro dessas políticas públicas de saúde, existem aquelas criadas diretamente para formação, desenvolvimento, distribuição, regulação e gestão dos profissionais de saúde, onde se tem fortificado a preocupação com a lógica da saúde prestada aos usuários. A fim de alcançar a consolidação de seus princípios, uma das estratégias difundidas pelo SUS foi a criação da Política Nacional de Educação Permanente em Saúde (PNEPS) ${ }^{3}$.

A PNEPS, desenvolvida a partir do ano de 2004 e reformulada em 2007, se insere no SUS com o objetivo de aproximar suas ações e serviços com as instituições formadoras, no sentido de promover mudanças na formação em saúde e provocar alterações nas práticas dominantes dos sistemas de saúde, por meio da problematização de suas próprias práticas e do trabalho em equipe ${ }^{3}$. Nesse sentido, pode ser compreendida como um dispositivo para transformação da área da saúde, em que ações são idealizadas em busca de uma mlehoria do padrão de assistência à saúde ${ }^{4}$.

Para isso, uma alternativa foi a criação da Residência Multiprofissional em Saúde (RMS), dispositivo de transformação das práticas da área da saúde, que busca proporcionar, na continuidade da formação dos profissionais, a qualificação para o trabalho voltado para o SUS 5 . O Ministério da Saúde propõe a estratégia da modalidade de Pós-Graduação - Lato Sensu com os programas de Residência Multiprofissional em Saúde, voltados para a educação em serviço destinada às diversas áreas, entre elas: Enfermagem, Farmácia, Fisioterapia, Fonoaudiologia, Medicina, Nutrição, Odontologia, Psicologia, Serviço Social e Terapia Ocupacional. Intenciona com a mesma, capacitar os profissionais a entenderem a multicausalidade dos processos mórbidos, individuais e coletivos, contextualizando o indivíduo em seu meio ambiente ${ }^{6}$.

Portanto, a Residência Multiprofissional em Saúde caracteriza-se pelo ensino e formação em serviço, e tem como objetivo promover a especialização de profissionais da saúde na promoção de atributos que possibilitem o exercício profissional com excelência nas áreas de cuidado integral à saúde, envolvendo as pessoas e as comunidades, da gestão e organização do trabalho e da educação na saúde, visando à melhoria da qualidade de vida ${ }^{6}$.

Do mesmo modo, essa modalidade de ensino em serviço, constitui-se como uma das estratégias potenciais para repensar o processo de formação em saúde, ao passo que assegura o desenvolvimento dos trabalhadores e instituições de saúde por meio de suas políticas implementadas ${ }^{7}$. Com a RMS, os residentes têm diversas oportunidades de aprimoramento teórico, teórico-prático e técnico, o que pode possibilitar a reflexão crítica de várias ações e aspectos, que emergem a partir das situações vividas durante esse processo de formação na Residência e da realidade no contexto da Saúde ${ }^{5}$. 
Para Bourget et al. ${ }^{8}$, designadamente a RMS com Área de Concentração em Saúde da Família, vem se mostrando ao longo desses anos, importante instrumento de formação e capacitação para os trabalhadores do setor Saúde. Ressaltam ainda, que é necessário manifestar que a atuação nos cenários peculiares e diferenciados que tangem esse programa é, nas vivências dos residentes, efetivos impulsionadores para a transformação das realidades.

Assim, a inserção dos residentes no serviço de saúde pode potencializar a construção de um processo de aprendizagem que passa a ter novas bases em sua constituição educacional, voltada ao desenvolvimento de atributos pessoais e profissionais que visam ao cuidado integral à saúde do sujeito e da comunidade ${ }^{8}$. A partir disso, e ao se considerar a importância e a necessidade de avaliação dos Programas de Residência Multiprofissional em Saúde pelos atores envolvidos no processo, este trabalho teve como objetivo analisar a percepção dos residentes de Saúde da Família acerca da estruturação do Programa de Residência Multiprofissional em Saúde da Universidade do Estado do Pará.

\section{Método}

O presente estudo foi do tipo observacional transversal, de caráter quantiqualitativo, com predomínio qualitativo. A pesquisa foi realizada nas dependências da Universidade do Estado do Pará, localizada município de Belém, no período de Outubro de 2016. Para a seleção da amostra, os critérios de inclusão da pesquisa consistiram em: ser residente integrante do Programa de Residência Multiprofissional em Saúde da Universidade do Estado do Pará; estar cursando o segundo ano e ser pertencente à Área de Concentração Saúde da Família. Sendo os critérios de exclusão: residente que não cursou o primeiro ano no Programa de Residência Multiprofissional em Saúde da Universidade do Estado do Pará. Assim, os participantes da pesquisa foram 10 dos 12 residentes integrantes do Programa de Residência Multiprofissional em Saúde da Universidade do Estado do Pará, sendo composta por: 4 (quatro) Fisioterapeutas, 3 (três) Terapeutas Ocupacionais e 3 (três) Enfermeiros.

Para a obtenção dos dados iniciais, inicialmente foi realizada a aplicação do questionário sócio demográfico, onde foram coletados dados acerca do perfil dos residentes entrevistados (sexo, idade, estado civil, profissão, tempo de formação, renda familiar e se possuiu outro vínculo empregatício). Posteriormente a esta etapa, utilizou-se com os residentes a entrevista semiestruturada, contendo cinco questões norteadoras, com o objetivo de obter os significados atribuídos pelos mesmos em relação aos aspectos que envolvem o Programa de Residência Multiprofissional em Saúde/UEPA. Os depoimentos foram gravados por meio digital de áudio e os discursos obtidos foram, posteriormente, transcritos na íntegra e devidamente categorizados para posterior interpretação do conteúdo. Com a finalidade de preservar a identidade dos residentes, os mesmos foram identificados por números, atribuídos aleatoriamente de acordo com a ordem de entrevista, como por exemplo R1, R2, R3, etc.

Para a análise dos dados, os resultados dos questionários provenientes da pesquisa quantitativa, foram digitados em um banco para execução de análise estatística descritiva, e posteriormente, para a construção de tabelas e gráficos no programa Excel $2007^{\circledR}$ (Microsoft). Os procedimentos para a pesquisa qualitativa se basearam, a partir de uma análise interpretativa das falas correspondentes ao conteúdo de cada pergunta do roteiro. Para esta análise, foi empregada a técnica de Análise de Conteúdo, constituída pelas etapas: pré-análise, exploração do material e tratamento dos resultados, inferência e interpretação ${ }^{9}$. Assim, após a transcrição, os dados foram codificados a partir das unidades de registro. Em seguida, como última etapa, realizou-se a categorização dos discursos, que consistiu na classificação dos elementos segundo suas semelhanças e diferenciações, com posterior reagrupamento em função 
de características comuns, gerando assim as categorias temáticas pertinentes aos dados obtidos.

O projeto foi aprovado pelo Comitê de Ética em Pesquisa da Universidade do Estado Pará (CEP/UEPA), parecer $n^{\circ}$ 1.754.665. Os residentes foram entrevistados, após concordância e assinatura do Termo de Consentimento Livre e Esclarecido.

\section{Resultados e discussão}

\section{Perfil dos entrevistados}

Quanto à caracterização do perfil dos residentes de Saúde da Família, a maioria era do sexo feminino $(70 \%, 7 / 10)$, onde se encontram na faixa etária entre 24 a 31 anos, tendo como estado civil predominante, solteiro $(70 \%, 7 / 10)$. Em relação à renda familiar, a maioria dos entrevistados $(80 \%, 8 / 10)$ alega possuir uma renda mensal acima de cinco salários mínimos. Sobre a naturalidade dos participantes, observou-se que a maioria dos residentes são naturais do Estado do Pará $(80 \%, 8 / 10)$, enquanto que metade destes, são oriundos do interior do Estado.

Com relação ao tempo de formação, a maioria dos entrevistados possui dois anos de formados $(80 \%, 8 / 10)$. Apenas dois residentes relataram possuir pós-graduação previamente ao ingresso no programa. Quanto ao vínculo empregatício anterior a Residência, o maior percentual de entrevistados afirmou não ter havido outro vínculo $(80 \%, 8 / 10)$. Observa-se assim, uma relação direta entre a idade, tempo de formação e vínculo empregatício, indicando que, em geral, a maioria dos profissionais do estudo tem a Residência Multiprofissional em Saúde, como primeira experiência profissional.

Ressalta-se, desse modo, uma relação direta entre o tempo de formação e a realização de pós-graduação, indicando que em geral, a maioria dos profissionais desta pesquisa tem a Residência Multiprofissional em Saúde, como uma forma de buscar também uma especialização. Vários autores consideram que atualmente, os programas de Residência são uma complementação à formação na graduação, tendo em vista as deficiências nela reconhecidas, assim como uma perspectiva de inserção no mercado de trabalho após a especializaçãó2.

\section{Análise das entrevistas}

Por meio da análise dos depoimentos dos residentes entrevistados, foi possível destacar cinco categorias de acordo com as questões norteadoras da entrevista:

\section{A Residência Multiprofissional em Saúde}

Na primeira categoria, que trata das percepções dos residentes sobre o significado da RMS, os depoimentos demonstraram a mesma, com uma das possibilidades de integração de ensino-serviço, com vistas ao desenvolvimento de competências profissionais necessárias para a consolidação do SUS:

[...] a Residência em si, tem de fato esse objetivo de treinar o profissional no serviço e formá-lo para o SUS (R4).

[...] eu entendo que a Residência foi criada para formar e qualificar para atender o SUS né? É uma estratégia excelente pra quem quer trabalhar no SUS, e ver o SUS andar de fato (R3).

Segundo Domingos ${ }^{10}$, o projeto político da RMS com área de concentração em Saúde da Família, como modalidade de pós-graduação, busca preparar, aperfeiçoar, atualizar e aprofundar os conhecimentos e a prática dos profissionais para atuarem na Estratégia Saúde da Família (ESF) e consequentemente, na Atenção Básica. Deste modo, através de qualificações como essa, se diminui o descompasso entre as políticas e projetos do SUS e os projetos pedagógicos das instituições de ensino. 
Eu acho que a Residência é uma estratégia do Sistema Único de Saúde, que visa à formação de profissionais para o SUS e pelo SUS, então você está tendo aquela oportunidade de se especializar pra trabalhar em um sistema que precisa muito de profissionais devidamente preparados (R1).

Diante da concepção sobre a especialização em serviço, a RMS foi entendida como uma contribuição para que profissionais se tornem um dispositivo de mudança do modelo de assistência à saúde na Atenção Básica no SUS. Salvador et al. ${ }^{11}$ propõe que se atue sobre as lacunas deixadas pelos espaços de formação, por meio de experiências que efetivamente problematizem esta formação.

[...] a Residência é uma especialização, voltada para formação em serviço, então a gente estuda e pratica, por isso a necessidade da teoria e prática estarem aliadas, e o residente, a partir daí, tem a função de modificar o serviço (R6).

Para Borges et al. ${ }^{12}$, a integração entre o trabalho e a educação passou a ser para $\mathrm{o}$ SUS uma forma de superar a dívida do sistema educacional com seus profissionais, melhorando em conjunto a qualidade da assistência prestada à população, sendo o treinamento em serviço é considerada a melhor técnica de aprendizado. Entretanto, a educação de profissionais de saúde deve gerar profissionais que não se restrinjam ao mero domínio da técnica, mas que sejam dotados de traços humanísticos, moralmente confiáveis, éticos e disciplinados ${ }^{13}$. Assim, é importante ressaltar a grande influência que as Instituições de Ensino Superior exercem na organização dos serviços de saúde, uma vez que estas instituições são responsáveis pela formação dos recursos humanos que irão atuar nestes serviços ${ }^{14}$.

O papel dos residentes

Quando questionados acerca da percepção sobre o seu papel na RMS, os entrevistados abordaram os seguintes entendimentos:

[...] eu to pra contribuir com a prestação de serviço que vai ser prestado a essa comunidade, mas eu também tô lá pra me especializar, é uma formação em serviço. Então, eu também to lá como uma pessoa que está em formação... eu me percebo assim (R1).

Eu me vejo... assim... como uma forma de melhorar a questão da assistência à saúde no meu local de prática e somar, no sentindo de aumentar a cobertura dessas demandas [...] a Residência tem um papel muito importante nesse sentindo de formação e ações de serviço [...] (R4).

Nesse sentido, Silva et al. ${ }^{15}$ ressaltam que o projeto pedagógico da RMS, assinala que se espera do residente a internalização de uma atitude crítico reflexiva, que se traduza em comprometimento com a viabilização de metodologias de atuação, pautada nos princípios do SUS, as quais visam a resolutividade das ações em saúde nos diferentes níveis de atenção do sistema.

Os programas elaborados com base na PNEPS, como é o caso das Residências Multiprofissionais, partem do pressuposto da aprendizagem significativa, por aproximar o mundo do trabalho ao mundo da educação, no qual o ambiente de aprendizagem é o próprio espaço dos serviços de saúde do SUS ${ }^{16}$. A partir dos relatos, foi possível perceber que a Residência, enquanto percepção de papéis, possibilitou uma maior reflexão desse profissional sobre o seu trabalho, a forma como é desenvolvido e sua influência em uma determinada realidade:

[...] a Residência em si nos dá essa visão, de que a gente tem um papel transformador dentro da nossa realidade, da realidade que a gente tá inserido [...] (R8).

[...] como um profissional recém formado, o programa da Residência me possibilitou conhecer melhor de perto a realidade, saber as condições de saúde e me especializar, melhorando a minha atuação né? como profissional mesmo [...] (R10). 
Atualmente, verifica-se uma preocupação crescente com as mudanças na formação profissional em saúde, na qual cada vez mais se configura a necessidade de profissionais aptos a contribuir com a sociedade num contexto de profundas mudanças não somente em seu próprio campo profissional ${ }^{17}$.

\section{A prática multiprofissional na residência}

As falas dos residentes também permitem observar a visão sobre o que é o trabalho multiprofissional no cenário da Residência:

A equipe multiprofissional ébem estabelecida entre os residentes, essa noção de equipe multiprofissional, de equipe em que cada um sabe suas próprias demandas, mas que compartilha juntos as experiências (R1).

[...] na residência eu me surpreendi muito, de forma positiva, com a atuação multiprofissional, porque a gente sai da graduação e a gente só conhece o que a nossa categoria faz, e aí você começa a olhar pro outro, você se sente maravilhado com aquilo: 'nossa a gente pode fazer isso juntos!' (R6).

Para Campos e Domitti ${ }^{18}$, a presença de diferentes formações profissionais, bem como um alto grau de articulação entre os profissionais é essencial, de forma que não só as ações sejam compartilhadas, mas também tenha lugar de processo interdisciplinar, no qual progressivamente os núcleos de competência profissionais específicos vão enriquecendo o campo comum de competências, ampliando, assim, a capacidade de cuidado de toda a equipe. No entanto, é possível observar, que quando se trata do trabalho realizado em conjunto com os profissionais dos serviços atuantes, emerge a dificuldade da prática multiprofissional:

[...] mas quando se parte para uma forma mais ampla de pensar, levando em consideração das equipes que nos acolhem, eu acho que essa questão da prática da multiprofissionalidade, ela ainda tá um pouco perdida (R1).

[...] falta uma percepção da equipe, sobre o a integração com o residente, do quanto a gente pode contribuir, exercer a prática multiprofissional com os residentes [...] (R9).

O cotidiano dos serviços de saúde é descrito por Salvador et al. ${ }^{11}$, como um entrave à atuação multidisciplinar da equipe de saúde, existindo divisões entre os diversos setores do saber, bem como entre os profissionais e a integralidade da atenção. Com frequência os profissionais da área da saúde não vivenciam, ao longo de sua formação, estratégias que articulem suas atividades e saberes com as de outros profissionais da equipe. Entende-se que embora exista um amplo reconhecimento sobre a pertinência da proposta do trabalho em equipe de saúde, sabe-se que este se constitui como um desafio ${ }^{19}$.

\section{Atividades curriculares da residência}

Sobre o conhecimento a cerca do projeto pedagógico da RMS, a maioria dos entrevistados relatou o desconhecimento a cerca deste:

Eu não conheço o projeto pedagógico da minha Residência, eu imagino que ele exista [...] mas esse projeto nunca chegou as minhas mãos e gostaria até de muito conhecer, porque seria fundamental conhecer. Temos contato com as disciplinas e no site, mas não com o todo (R1).

[...] porque é assim né?... os residentes chegam, nos é apresentado às normas, mas o nosso projeto pedagógico eu não conheço, há não ser as disciplinas mesmo (R5).

Apesar da pouca clareza demonstrada pelos residentes em relação às propostas pedagógicas da RMS, observa-se que os mesmos se apresentam atentos às formas como estes processos de formação se desenvolvem, sinalizando para um distanciamento da teoria das disciplinas com a prática vivenciada: 
Você tá tendo uma prática e tendo outra teoria. Apesar que a nossa teoria é gratificante, você passar por todos aqueles módulos, mas eles seriam mais ricos se fosse trabalhado ao mesmo tempo. Acho até que as disciplinas contemplam a minha prática (R2).

[...] a gente vê as disciplinas depois que a gente passou pelo local de prática, eu acho que a gente perde a oportunidade de acrescentar nos serviços, porque isso também é o papel da residência né? (R6).

As falas também evidenciaram como parte das questões problematizadas, uma ideia de prejuízo aos residentes referente à questão teórica do curso, pois essas foram apontadas como dissociadas das vivências nos cenários de prática atuantes.

[...] foram poucos momentos que a gente conseguiu colocar a teoria na prática, então a teoria ficou muito fechada, se distanciou muito (R10).

Barros $^{20}$ contribui com seu pensamento ao afirmar que os processos de formação "[...] têm sido analisados como desvinculados e descomprometidos com a realidade, não produzindo saberes que revelem ou transformem essa realidade" (p.138). É importante reforçar que a estruturação e organização dos conteúdos teóricos da Residência devam contribuir na ampliação da visão de saúde dos residentes, mediante as realidades observadas nos espaços de atuação, vinculando e reafirmando que teorias e práticas são instâncias indissociáveis ${ }^{21}$.

\section{A atuação dos preceptores}

O preceptor é o profissional experiente e atuante no serviço de saúde, com título de especialista na área de formação, que apresenta além da competência e habilidade clínica, a capacidade para conduzir o residente no processo de aprender, estimulando e possibilitando o desenvolvimento pessoal e profissional do mesmo. Portanto, "[...] compete ao preceptor orientar e avaliar o residente, atuando também como guia e modelo [...]"21 (p.12). Entretanto, uma abordagem de destaque nas falas dos residentes se referiu ao pouco ou nenhum conhecimento a cerca do papel do preceptor ou a atuação dos residentes na RMS:

Eu acho que o preceptor, na prática da residência, é um profissional que está completamente perdido, mas às vezes que até os próprios residentes [...] são pessoas pra quem foram dadas uma missão, mas sem nenhum tipo de capacitação, de orientação ou nenhum tipo de preparo (R1).

[...] o que a gente observa muitas vezes, é que o preceptor não sabe o que é ser preceptor [...] a gente precisa explicar pra eles, dentro dos nossos conhecimentos o que é a Residência (R6).

Contudo, historicamente não há exigência de formação docente para o exercício da preceptoria, não havendo definição de requisitos mínimos necessários nem avaliação desses atributos. O preceptor é muito exigido, mas, em geral, há poucos ou nenhum programa para capacitação, qualificação, formação pedagógica e didática, nem remuneração diferenciada para esses profissionais, uma vez que se acredita que, para ser preceptor, basta ser um bom profissional de saúde ${ }^{13}$. A maior parte dos preceptores da RMS são profissionais que já desempenham atividades assistenciais em Saúde da Família, cujas competências e experiência prática os levaram a ser convidados pela coordenação do Programa para compor o grupo de preceptoria ${ }^{22}$.

Eles não tiveram oportunidade de se preparar para exercer essa função, aí às vezes ficam perdidos com a questão de supervisionar, até onde ele pode, até onde não pode, com a questão de mandar [...] (R1).

Os preceptores não têm a capacitação pra ser realmente um preceptor, a gente chega, e eu percebo que eles não têm um conhecimento do que é ser um preceptor. Eles acham que a residência é... como eu posso dizer? Um estagiário, mão de obra pra eles, então acho que ainda falta capacitação (R2).

Rocha e Ribeiro ${ }^{23}$ pontuam que o profissional que atua como preceptor ou professor universitário necessita de capacitação específica, não podendo restringir a formação 
a um diploma de bacharel, um doutorado ou mesmo ao fato de atuar como bom profissional na área em questão. Para tanto, é necessário que a relação entre estes trabalhadores e residentes seja mediada pelo processo de ensino-aprendizagem, o que requer, sobretudo, uma relação horizontalizada de troca, bem como ações institucionais que qualifiquem essa atividade pedagógica. Este resultado corrobora o entendimento da RMS como uma proposta que caminha no sentido da mudança da formação do trabalhador, voltada para integração ensino-serviço ${ }^{21}$. Notou-se ainda, uma crítica dos residentes da RMS, a respeito da pouca participação e interação de preceptores e coordenador sobre os rumos do processo de formação dos residentes, como exposto pelo participante:

\section{[...] de modo geral, sinto falta dessa união entre coordenação e preceptores (R6).}

A comunicação e o planejamento inadequados entre os coordenadores de programas de residência e os preceptores, levam a falsas conclusões quanto aos objetivos de ensino, que nem sempre são os mesmos entre preceptores e programas. É fundamental que os coordenadores se assegurem de que a prática de ensino dos docentes atinja os objetivos dos projetos pedagógicos dos cursos de graduação e dos programas de Residência ${ }^{24}$.

\section{Considerações finais}

Ao analisar o Programa de Residência Multiprofissional em Saúde procurou-se obter informações dessa, sob a visão de profissionais pertencentes à Estratégia Saúde da Família, sendo enfatizada a atuação profissional e vivências de cada residente, dentro desse contexto. Ressalva-se que em suma, os principais aspectos considerados assertivos foram observados em relação à possibilidade de formação/aperfeiçoamento profissional na assistência à saúde, a possibilidade de transformar a realidade nos serviços atuantes, os conhecimentos adquiridos através das teorias e práticas ofertadas, o entendimento da funcionalidade do SUS a partir da Atenção Básica e a possibilidade de exercer a prática multiprofissional. Por outro lado, os aspectos desfavoráveis mais evidentes foram identificados como o desconhecimento acerca do projeto pedagógico; a forma incorreta como o residente é visto e o desconhecimento de suas funções; o despreparo do preceptor enquanto peça fundamental da Residência; a fragilidade de uma formação a partir da teoria e prática ofertada; e além dos desafios enfrentados na prática multiprofissional. Embora alguns problemas possam ser identificados no programa de RMS, é importante destacar que o desejo de cada entrevistado, para um melhor aproveitamento do aprendizado é, de fato imprescindível, como foi possível destacar nas falas dos residentes. Essa questão leva à reflexão, por exemplo, dos motivos revelados pelos residentes em optar pelo curso de especialização em Saúde da Família, como a oportunidade de mais experiências práticas na Atenção Básica, o que atende as necessidades por eles percebidas em relação à aquisição de competências profissionais. Os resultados obtidos no presente trabalho permitiram concluir que, o estudo oportunizou aos residentes de Saúde da Família, analisar seu contexto de trabalho, demarcando uma particularidade deste processo de ensino que visa à formação de um profissional crítico, que saiba atentar-se a realidade e possa contribuir na implementação de mudanças necessárias para consolidação de um serviço do SUS, como são as residências multiprofissionais. Por fim, destaca-se que neste trabalho foram ouvidos apenas os residentes, e se ressalta a necessidade de outras pesquisas avaliativas que abordem os demais atores envolvidos como tutores, preceptores, profissionais dos serviços e a coordenação do programa. 


\section{Referências}

1. Brasil. Conselho Nacional de Secretários de Saúde. Sistema Único de Saúde. Brasília: CONASS; 2011.

2. Nascimento D, Oliveira MA. Reflexões sobre as competências profissionais para o processo de trabalho nos Núcleos de Apoio à Saúde da Família. Mundo Saude. 2010;1(1):92-6. http://dx.doi.org/10.15343/0104-7809.201019296.

3. Brasil. Ministério da Saúde. Secretaria de Gestão do Trabalho e da Educação na Saúde. Departamento de Gestão da Educação em Saúde. Política Nacional de Educação Permanente em Saúde. Brasília: Ministério da Saúde; 2009. 64 p.

4. Lino MM, Backes VMS, Ferraz F, et al. Educação permanente dos serviços públicos de saúde de Florianópolis, Santa Catarina. Trab Educ Saúde. 2009;7(1):115-36.

5. Lobato CP. Formação dos trabalhadores de saúde na Residência Multiprofissional em Saúde da Família: uma cartografia da dimensão política [tese]. Londrina (PR): Universidade Estadual de Londrina; 2010. 117 p.

6. Silva JC, Contim D, Ohl RIB, et al. Percepção dos residentes sobre sua atuação no programa de residência multiprofissional. Acta Paul Enferm. 2013;28(2):132-8. http://dx.doi.org/10.1590/1982-0194201500023.

7. Brasil. Ministério da Saúde. Secretaria de Atenção à Saúde. Departamento de Atenção Básica. Política Nacional de Atenção Básica. Brasília: Ministério da Saúde; 2012.

8. Bourget MMM, Ghedin R, Vanz L, et al. Residência Multiprofissional em Saúde da Família: a experiência da Faculdade e Casa de Saúde Santa Marcelina In: Brasil. Residência multiprofissional em saúde: experiências, avanços e desafios. Brasília: Ministério da Saúde; 2006.

9. Bardin L. Análise de conteúdo. 7.ed. Portugal: Geográfica; 2009.

10. Domingos CM. A Residência Multiprofissional em Saúde da Família: o olhar do trabalhador de saúde. [dissertação]. Londrina (PR): Programa de Pós-graduação em Saúde Coletiva, Centro de Ciências da Saúde, Universidade Estadual de Londrina; 2012.

11. Salvador AS, Medeiros CS, Cavalcanti PB, Carvalho RN. Construindo a multiprofissionalidade: um olhar sobre a residência multiprofissional em Saúde da Família e Comunidade. Rev Bras Cien Sau. 2011;15(3):329-38. http://dx.doi.org/10.4034/ RBCS.2011.15.03.08.

12. Borges FT, Garbin CAS, Siqueira CE, et al. Escolas técnicas do SUS (ETSUS) no Brasil: regulação da integração ensino serviço e sustentabilidade administrativa. Ciênc Saúde Coletiva. 2012;17(4):977-87.

13. Santos EG, Mannarino RR, Leher VL, Teixeira EM, Goldwasser RS, Bravo GP No. Avaliação da preceptoria na residência médica em cirurgia geral, no centro cirúrgico, comparação entre um hospital universitário e um hospital não universitário. Rev Col Bras Cir. 2012;39(6):547-52. http://dx.doi.org/10.1590/S0100-69912012000600017. PMid:23348654.

14. Almeida S. A formação do fisioterapeuta sob a perspectiva da integralidade [dissertação]. Brasília (DF): Programa de Pósgraduação em Processos de Desenvolvimento Humano e Saúde, Instituto de Psicologia, Universidade de Brasília; 2013. $108 \mathrm{p}$.

15. Silva CT, Terra MG, Kruse MHL, Camponogara S, Xavier MS. Residência multiprofissional como espaço intercessor para a educação permanente em saúde. Texto Contexto Enferm. 2016;25(1):1-9.

16. Lopes SES, Piovesan ETA, Melo MO, Pereira MF. Potencialidades da educação permanente para a transformação das práticas em saúde. Com Ciênc Saúde (Porto Alegre). 2007;18:147-55.

17. Ferreira R, Fiorini V, Crivelaro E. Formação profissional no SUS: o papel da atenção básica em saúde na perspectiva docente. Rev Bras Educ Med. 2010;2(2):207-15. http://dx.doi.org/10.1590/S0100-55022010000200004.

18. Campos GWS, Domitti AC. Apoio matricial e equipe de referência: uma metodologia para gestão do trabalho interdisciplinar em saúde. Cad Saude Publica. 2007;23(2):399-407. http://dx.doi.org/10.1590/S0102-311X2007000200016. PMid:17221089.

19. Albuquerque VS, Gomes AP, Rezende CHA, Sampaio MX, Dias OV, Lugarinho RM. A integração ensino-serviço no contexto dos processos de mudança na formação superior dos profissionais da saúde. Rev Bras Educ Med. 2008;32(3):356-62. http:// dx.doi.org/10.1590/S0100-55022008000300010.

20. Barros MEB. Desafios ético-políticos para a formação dos profissionais de saúde: transdisciplinaridade e integralidade. In: Pinheiro R, Mattos R, Ceccim R, organizadores. Ensinar saúde: a integralidade e o SUS nos cursos de graduação na área da saúde. Rio de Janeiro: Cepesc; 2006. p. 131-151.

21. Shikasho L. Os Programas de Residência e a Integralidade da Atenção: um estudo sobre a micropolítica do trabalho e da formação em saúde [dissertação]. Juiz de Fora (MG): Universidade Federal de Juiz de Fora; 2013. 209 p.

22. Nascimento DDG, Quevedo MP. Aprender fazendo: consideração sobre a Residência Multiprofissional em Saúde da Família na qualificação de profissionais de Saúde. In: Bourget MMM, organizador. Estratégia Saúde da Família: a experiência da Equipe de Reabilitação. São Paulo: Martinari; 2008. p. 43-59.

23. Rocha H, Ribeiro V. Curso de formação pedagógica para preceptores do internato médico. Rev Bras Educ Med. 2012;36(3):34350. http://dx.doi.org/10.1590/S0100-55022012000500008.

24. Dias IMÁV, Devincenzi MU, Silva ER, Uchôa-Figueiredo LR, Thomaz SMT. Residência multiprofissional em saúde e suas normativas. In: Uchôa-Figueiredo LR, Rodrigues TF, Dias IMAV. Percursos interprofissionais: formação em serviços no Programa Residência Multiprofissional em Atenção à Saúde. Porto Alegre: Editora Rede Unida; 2016. p. 27-45. (Série Vivências em Educação na Saúde).

\section{Contribuição dos autores}

Ana Paula Nascimento de Lima e Biatriz Araújo Cardoso, participaram de todas as etapas da pesquisa e confeccção do manuscrito. Ila Iandara Araújo de Souza e Lays Ariane Teixeira dos Santos contribuíram com as análises dos dados e confecção do manuscrito. 